\title{
ÍNDICE DE DESEMPEÑO INNOVADOR EN LOS SUBSECTORES INDUSTRIALES COLOMBIANOS ENTRE 1996 Y 2005*
}

\author{
JORGE ALONSO MANRIQUE HENAO**, JORGE ROBLEDO VELÁSQUEZ*** \& ÁLVARO LEMA TAPIAS***** \\ UNIVERSIDAD PONTIFICIA BOLIVARIANA - MEDELLÍN
}

Recibido/ Received/ Recebido: 24 /09/2013 - Aceptado/ Accepted / Aprovado: 29/05/2014

\begin{abstract}
Resumen
En diferentes países se han calculado índices a partir de encuestas de innovación bajo especificaciones planteadas desde el Manual de Oslo de la OCDE. No obstante, las características de la información existente en las realizadas en Colombia en los años 1996 y 2005 no permiten la comparabilidad directa de las variables. Esto llevó a proponer un nuevo índice de desempeño innovador de la industria basado en la metodología del índice de valor de importancia relativizado, utilizado en otros ámbitos como las clasificaciones etnobotánicas. Esta metodología, permite visualizar el fenómeno en los dos momentos de las encuestas realizadas en Colombia y se revela como una herramienta para relacionarlas. Se encontró que el desempeño innovador industrial en el año 1996 fue caracterizado principalmente por la innovación de mercado y organizacional, mientras que en 2005, su principal característica fue la innovación potencial.
\end{abstract}

Palabras clave: Índice de desempeño innovador, Índice de valor de importancia, Encuestas de innovación, Subsectores industriales, Innovación industrial.

\section{INNOVATION PERFORMANCE INDEX IN COLOMBIAN INDUSTRIAL SUBSECTORS BETWEEN 1996 AND 2005}

\begin{abstract}
Rates are calculated in different countries from innovation surveys raised under specifications from OECD's Oslo manual. Nevertheless, information characteristics do not allow direct comparability of the variables in Colombia during 1996 and 2005. This led to propose a new innovation performance index of industry based on importance value index methodology relativized and used in other areas such as ethno-botanical classifications. This methodology allows visualizing the phenomenon in both
\end{abstract}

\footnotetext{
Artículo de investigación enmarcado en la tesis doctoral presentada en 2012 denominada "El uso energético y la innovación en la industria colombiana a partir de encuestas oficiales", cofinanciada por la Universidad Pontificia Bolivariana y Colciencias, y a través de la cual los autores tuvieron acceso a los datos de las dos primeras encuestas de innovación realizadas en Colombia en los años 1996 y 2005 para el cálculo de los indicadores que se describen en este artículo.

Los autores agradecen a Colciencias por la financiación del proyecto "Descubrimiento de conocimiento sobre la innovación en Colombia a partir de bases de datos", del cual formó parte la investigación aquí reportada, y a la Universidad Pontifica Bolivariana y a la Universidad Nacional de Colombia por la cofinanciación del proyecto y el apoyo brindado a los autores para su participación en el mismo.

** Doctor en Ingeniería, Magíster en Gestión Tecnológica, Docente investigador del Grupo de Investigación y Posgrados en Gestión de la Tecnología y la Innovación GTI de la Universidad Pontificia Bolivariana Sede Medellín, Colombia. Correo electrónico: jorge.manrique@upb.edu.co. Tel: (57) 4- 3544522. Dirección postal: Circular 1 \# 70-01, bloque 11, Universidad Pontificia Bolivariana, Medellín.

**** Doctor en Estudios de Política Científica y Tecnológica de SPRU - Universtiy of Sussex. Profesor de la Facultad de Minas, Universidad Nacional de Colombia - Sede Medellín. Correo electrónico: jrobledov@unal.edu.co. Tel: (57) 4-4255227. Dirección postal: Carrera 80 \# 65-223, Medellín.

***** Docente Investigador del Posgrado de Bosques y Conservación Ambiental, Universidad Nacional de Colombia Sede Medellín, Colombia. Correo electrónico: adjlema@unal.edu.co. Tel: (57) 4-2386051. Dirección postal: Postgrado de Bosques y Conservación Ambiental, Universidad Nacional, Autopista norte, Carabineros, Medellín.
} 
surveys conducted in Colombia and it is considered as a tool to correlate them. It was found that innovation industrial performance in 1996 was characterized mainly by the market and organizational innovation, while in 2005 its main feature was the potential innovation.

Keywords: Innovation performance index, Importance value index, Innovation surveys, Industrial subsectors, Industrial innovation.

\title{
ÍNDICE DE DESEMPENHO INOVADOR NOS SUBSETORES INDUSTRIAIS COLOMBIANOS ENTRE 1996 E 2005
}

\begin{abstract}
Resumo
Em diferentes países se calculam índices de inovação a partir de enquetes com especificações propostas desde o Manual de Oslo da OCDE. Apesar disto, as características da informação existente nos levantamentos realizados na Colômbia nos anos 1996 e 2005 não permitem a comparação direta das variáveis. Isto levou a propor um novo índice de desempenho inovador da indústria baseado na metodologia do índice de valor de importância relativizado utilizado em outros âmbitos como as classificações etnobotânicas. Esta metodologia permite visualizar o fenômeno nos dois momentos das pesquisas realizadas na Colômbia e se revela como uma ferramenta para relacioná-las. Encontrou-se que o desempenho inovador industrial no ano 1996 foi caracterizado principalmente pela inovação de mercado e organização enquanto que em 2005 sua principal característica foi a inovação potencial

Palavras chave: Índice de desempenho inovador, Índice de valor de importância, Enquetes de inovação, Subsetores industriais, Inovação industrial.

Manrique, J., Robledo, J. \& Lema, A. (2014) Índice de desempeño innovador en los subsectores industriales colombianos entre 1996 y 2005. En: Revista de la Facultad de Ciencias Económicas de la Universidad Militar Nueva Granada. rev.fac.cienc.econ, XXII(2).

JEL: O31, O32, O39.
\end{abstract}

\section{Introducción}

Durante las últimas décadas, la posibilidad de acceder a grandes cantidades de información en los países desarrollados ha permitido la formulación de metodologías y modelos de análisis de la innovación, cuyos resultados son insumo para la formulación de políticas públicas y estrategias empresariales; situación que está lejos de replicarse en los países en desarrollo. Las actuales discusiones sobre la competitividad tecnológica referentes a la habilidad para introducir un nuevo bien o servicio a los mercados internacionales, remiten a la capacidad de absorción de conocimiento y la innovación de un país (Fagerberg, Srholec \& Knell, 2007) dado que, el conocimiento resultado de la investigación constituye el requisito de ingreso a la sociedad y la economía del conocimiento. Por otra parte, se entiende la innovación como el fenómeno que explica la producción y transformación del conocimiento científico y tecnológico en riqueza económica, bienestar social y desarrollo humano.

El marco teórico y metodológico para la medición y análisis de la innovación se basa en el Manual de Oslo (OECD, 2005) que propone, entre otras cosas, las pautas para elaboración de encuestas nacionales de innovación, a partir de las cuales se ha planteado una serie de indicadores de innovación. No obstante, su construcción e interpretación son particulares $y$ dependen de las fuentes utilizadas, por lo cual se afirma la necesidad de disponer de un amplio conjunto de indicadores que se refuercen y complementen entre sí. 
En los países en desarrollo sin embargo, existen dificultades con la generación, aplicación y análisis de encuestas que no permiten construir indicadores ni efectuar análisis como los ya mencionados. Desde esta perspectiva, es importante establecer la posibilidad de iniciar estudios sobre indicadores de innovación que permitan aportar nuevos procedimientos $y$ modelos de análisis de datos, para establecer relaciones importantes que puedan arrojar resultados sobre el desempeño innovador en los subsectores y grupos industriales, que contribuyan a la correcta formulación de políticas que favorezcan la competitividad de la industria nacional. Se propone entonces, la construcción de un indicador que considere las limitaciones de la información y datos no comparables de dos encuestas de innovación colombianas analizadas, y la eliminación de co-linealidades entre las variables consideradas, característica poco analizada en los índices existentes.

\section{Problema de investigación}

Las actuales discusiones sobre la competitividad de la industria remiten a la capacidad de absorción de conocimiento y la capacidad de innovación. Respecto a la innovación, el Manual de Oslo (OECD, 2005) constituye un referente para la recolección de datos y la construcción de indicadores, insumo muy importante en la discusión y análisis de los fenómenos relacionados con la creación del conocimiento y su transformación en riqueza, bienestar y desarrollo. Previamente, se había planteado también el Manual de Bogotá (RICYT, OEA, CYTED, COLCIENCIAS \& OCYT, 2001) como referente de normalización para actividades de innovación en América Latina y el Caribe. No obstante, aunque estos manuales han inspirado diferentes indicadores, su interpretación se ha dejado a cada país ocasionando que hasta ahora, las encuestas diseñadas y los indicadores derivados difieran significativamente (Lugones \& Suarez, 2010).

En Colombia no se hacen encuestas exclusivas para empresas innovadoras, ni existe aún la discriminación de información entre empresas innovadoras y empresas no innovadoras, como sí ocurre en los países de la OCDE lo cual, impone una condición de entrada para el proceso de análisis. Malaver \& Var- gas (2006) establecen que, varios estudios realizados en Colombia sobre el comportamiento innovador y sobre el desarrollo de sus capacidades tecnológicas, han mostrado que la tecnología y la innovación no desempeñan un papel estratégico, sino funcional y de apoyo a las estrategias de modernización y competitividad de las empresas; que los procesos de innovación son reactivos y episódicos, y que la gestión tecnológica es de carácter informal. Ante esta situación los diferentes estudios que además, son de carácter puntual, deben ser validados a través de otros análisis.

Una de las condiciones importantes para reducir la brecha de conocimiento es generar información pertinente y facilitar el acceso a microdata, asunto ya resuelto en gran medida en varios de los países de la OCDE pero, en Colombia, aun es un problema por resolver, con impactos importantes en la cantidad y calidad de la investigación y el conocimiento producido a partir de los resultados de las encuestas oficiales, con reportes previos de hasta $26,8 \%$ como cifra de pérdida de información en la primera Encuesta de Innovación EDT1 realizada en 1996 (Salazar \& Vargas, 1998). Adicionalmente, las bases de datos de las encuestas EDT1 y de la Segunda Encuesta de Innovación EDT2 realizada en 2005, presentaban gran cantidad de errores por incumplimiento de normas de diligenciamiento de formularios como datos: por fuera de rango, mal registrados, o con información no disponible, entre otros, mientras que, el Manual Base suministrado con esta encuesta, presentaba errores en la identificación de algunas variables. Además, luego de su procesamiento, se encontró alta redundancia en la información de las variables en ambas encuestas (Manrique, 2012).

Debido a la complejidad de la medición y análisis del fenómeno en la actualidad, se afirma la necesidad de disponer de un amplio conjunto de indicadores que se refuercen y complementen entre sí (Buesa et al., 2001).

Puesto que, en Colombia se han realizado algunas encuestas de innovación no analizadas desde la perspectiva de los índices de innovación propuestos en la literatura y calculados regularmente, luego de analizar algunas de las limitaciones de sus datos, se 
propone la construcción de un índice que permita incrementar el conocimiento sobre las dinámicas empresariales, sectoriales y regionales con respecto al desempeño innovador de los subsectores industriales colombianos en el período de realización de las encuestas, con una metodología que, desde la estadística multivariada considere las limitaciones y la eliminación de colinealidades o redundancia de información en las variables seleccionadas. Particularmente, esta situación tampoco se ha considerado en la selección de variables para el cálculo de los indicadores existentes (Manrique, 2012).

\section{Marco teórico}

La posibilidad de acceder a gran cantidad de información sobre aspectos relacionados con la innovación en los países desarrollados, y el desarrollo de nuevas metodologías de análisis han incentivado desde hace varias décadas las propuestas tendientes a revisar las diferentes aproximaciones para formular modelos empíricos y sintéticos; esto se hace actualmente con la ayuda de herramientas estadísticas y econométricas por la gran cantidad de variables necesarias para identificar grupos de capacidades que son críticas para la apropiación eficiente del conocimiento o Catching up (Fagerberg \& Srholec, 2005).

El marco conceptual y metodológico actual para la medición y análisis de la innovación mediante encuestas, se basa en los planteamientos del manual de Oslo (OECD, 2005), el cual se fundamenta, a su vez, en diferentes teorías y resultados de aplicaciones empíricas en países de la OCDE, el cual también hace recomendaciones para abordar el tema en países subdesarrollados. No obstante, aunque existe la posibilidad de realizar comparaciones de resultados entre países, se reconoce que no todos los planteamientos hechos pueden aplicarse de igual manera en los países de la OCDE y en el conjunto no homogéneo de aquellos en vías de desarrollo pues, el inicio de la recolección y sistematización de datos es mucho más reciente y existen limitaciones con el diseño de las encuestas de innovación, la regularidad de su aplicación y la confiabilidad de la información recolectada, como se evidenció durante el desarrollo de este trabajo.
Los diferentes tipos de innovación se fundamentan en las actividades innovadoras desarrolladas por la empresa, las cuales se corresponden con todas las operaciones científicas, tecnológicas, organizativas, financieras y comerciales que conducen efectivamente a la introducción de innovaciones clasificadas en cuatro categorías: innovación de productos, de procesos, de organización y de mercadotecnia. Las empresas que innovan en cualquiera de estas categorías durante el período bajo análisis, se reconocen como innovadoras. Se introduce, además, la categoría de empresas potencialmente innovadoras, como aquellas que hicieron esfuerzos invirtiendo en alguna actividad de innovación y desarrollo tecnológico, pero la consecución de los objetivos se encuentra en proceso, fueron abandonados o no aplican (OECD, 2005). La existencia de un gran número de empresas potencialmente innovadoras puede sugerir la presencia de serias barreras a la innovación o de tentativas prematuras al respecto con ausencia de los recursos necesarios. Desde la perspectiva de su estimación y análisis, los esfuerzos realizados por las empresas y las organizaciones (actividades innovadoras) y las capacidades (existencias y flujo) son, incluso más importantes, que los resultados (innovaciones).

La discusión está abierta y se encuentran propuestas para la medición de la innovación desde diferentes frentes, taxonomías y metodologías, como los propuestos por Archibugi \& Pianta (1996); Reichstein (2004); Buesa, Heijs, Martínez Pellitero \& Baumert (2006); Olaya (2009); Delgado \& Sánchez (1999); Pedraza et al. (2008); He \& Fallah (2011); Okay, Konukman \& Akman (2009); Sanchez \& Castrillo (2006); Green, Gavin \& Aiman-Smith (1995); Blackman, Seligman \& Sogliero (1973); Leonardi, García Casal \& Cristiano (2009), entre muchos otros, lo cual muestra la complejidad que enfrenta el fenómeno y la dificultad de su medición.

En Colombia, por su parte, en las encuesta de innovación no se diferencian las empresas innovadoras de las no innovadoras, lo cual impone una condición de entrada para el proceso de análisis, como si se da en los países de la OCDE. Malaver \& Vargas (2006) establecen que varios estudios realizados en Colombia sobre el comportamiento innovador y 
sobre el desarrollo de sus capacidades tecnológicas, han mostrado que la tecnología y la innovación no desempeñan un papel estratégico, sino funcional y de apoyo a la modernización y competitividad de las empresas; que los procesos de innovación son reactivos y episódicos, y que la gestión tecnológica es de carácter informal.

Ante esta situación, los diferentes estudios que, además, son de carácter puntual, deben ser validados a través de otros análisis. Entre estos están el de Robledo (1997), quien estudia el papel de las universidades y el gobierno en el proceso de innovación industrial; el realizado por Mora Luna \& Davidsen (2006), quienes estudian el bajo rendimiento de la innovación desde la dinámica de sistemas; el trabajo de Langebaek \& Velásquez (2007) que analiza los bajos niveles de $\mathrm{I}+\mathrm{D}$ encontrados en la Encuesta de Innovación de 2004-2005 realizada en Colombia. Además está el documento de Chaves Castro (2005), quien hace algunos análisis de la innovación desde la producción y las importaciones de materias primas; y por última está el trabajo de Cardona \& Cano (2005), que concluye que el sistema económico colombiano no produce información que propicie difusión tecnológica, apropiación del conocimiento e innovación competitiva, entre otros.

\section{1 Índices de innovación}

Durante las últimas décadas se ha demostrado la importancia de la innovación tecnológica y organizativa como un factor clave en el desarrollo y el crecimiento económico. La medición de su impacto en las actividades económicas, se ha convertido en un aspecto central para el diseño y aplicación de las políticas públicas y estrategias privadas, por lo que la creación de indicadores que puedan identificar las especificidades regionales y nacionales, y compararse internacionalmente, sea uno de los puntos de debate más importantes (Lugones \& Suarez, 2010). Dos de los principales exponentes de esta necesidad son el Manual de Oslo (OECD, 2005) y el de Bogotá (RICYT, OEA, CYTED, COLCIENCIAS \& OCYT, 2001).

Las mediciones de la innovación presentan dificultades en la aplicación y en las estadísticas intrínsecas o solo reflejan alguno de los aspectos de la innovación que han tratado de subsanarse, en parte, desde las encuestas de innovación (Molero, 2007). De ahí, que los diferentes tipos de indicadores se han desarrollado en condiciones particulares que obedecen a la disponibilidad, regularidad y calidad de los datos, o la posibilidad de aplicación de metodologías econométricas o estadísticas, limitaciones que restringen su utilidad y, por tanto, para el análisis de los fenómenos relacionados con la creación del conocimiento científico y tecnológico. Adicionalmente, su interpretación se ha dejado a cada país, ocasionando que, hasta ahora, las encuestas diseñadas y los indicadores derivados difieran significativamente (Lugones \& Suarez, 2010). Se afirma entonces, la necesidad de disponer de un amplio conjunto de indicadores que se refuercen y complementen entre sí (Buesa, Navarro, Molero, Aranguren \& Olarte, 2001).

Dentro de los índices considerados se encuentran propuestas que tratan de medir las capacidades tecnológicas, de innovación o de otros aspectos relacionados desde diferentes frentes, metodologías y fuentes utilizadas, como el índice de tecnología $\mathrm{ArCo}$ propuesto por Archibugi \& Coco (2004), que mide las capacidades tecnológicas de un país; el Índice de Innovación en las Regiones Españolas de Martínez \& Baumert (2003); el Índice de Innovación Global GII propuesto por Dutta et al. (2008); el Índice de Desempeño Innovador IDI calculado para definir el desempeño innovador un grupo de firmas agroindustriales argentinas y los factores significativos que pudieran explicarlo (Leonardi, García \& Cristiano, 2009); el Índice de Innovación II propuesto en Centroamérica por el International Development Research Centre IDRC para las PyMEs en varios países, con base en un índice propuesto por el Networked Readiness Index -NRI-, con el fin de identificar el impacto de la adopción de las tecnologías de la información y la comunicación en el desempeño de las empresas, entre otros. Una aproximación al cálculo de índices de innovación para la industria colombiana con base en este índice de innovación II se encuentra en Manrique \& Velázquez (2012) quienes concluyen que, en el año 1996 los diferentes subsectores industriales colombianos tenían más actividades de innovación que las realizadas para el año 2005 y evidencian la existencia de limitaciones importantes en la informa- 
ción disponible para su construcción. No obstante la cantidad de índices elaborados para abordar los procesos y factores importantes en el entendimiento de la innovación en la industria, en el presente estudio nuevamente se evidencian las limitaciones de información que dificultan el cálculo de un índice para el caso colombiano con las características y elementos de comparación internacional.

\subsection{Las fuentes de datos}

Los datos utilizados al momento de iniciar el presente estudio provienen de dos fuentes disponibles. La primera la Encuesta sobre Desarrollo Tecnológico en el Establecimiento Industrial Colombiano (EDT1) aplicada en 1996, por el Departamento Nacional de Planeación (DNP) y Colciencias, a una muestra de 885 establecimientos del sector manufacturero (excluyendo el sector tabaco), de un universo de 4.501 establecimientos y, la Segunda Encuesta de Innovación y Desarrollo Tecnológico (EDT2), aplicada en 2005 a una muestra de 6.213 empresas, aunque, según (Restrepo, 2008), con datos útiles solo para 4.279 empresas.

Entre muchas diferencias tanto en el diseño, como en la aplicación y en la recolección de datos en ambas encuestas, el criterio para definir el tamaño de las empresas, es uno de ellos. Para efectos de comparación de las encuestas en el presente estudio, se consideró la clasificación que se hizo en la EDT1 y que permite diferenciar empresas entre micro, pequeñas, medianas y grandes, según el número de sus empleados así, A: entre 20 y 49; B: entre 50 y 99; C: entre 100 y 199; y D: 200 y más. Además de las diferencias estructurales en ambas encuestas, también presentan características y errores de diligenciamiento (Manrique, 2012), que obligó a la eliminación de variables importantes para el estudio. Por esta razón se propone un método para su relacionamiento.

Dados los niveles de agregación de los datos de las Encuestas de Innovación de 1996 y 2005, la unidad de análisis para la investigación corresponde a un nivel de agregación según clases CIIU a 2 dígitos, que se identifican como Alimentos, bebidas y tabaco: AlimByTb; Textiles, confecciones y cuero: TxtConfCr; Madera y sus productos:MadPrdts; Papel e im- prentas: PapelImpr; Químicos, caucho y derivados del petróleo: Qcos; Minerales no metálicos: Minometal; Metales básicos: MetalBas; Productos metálicos, maquinaria y equipos: Pdtmetalymaq.

\subsection{Propuesta del Índice de Desempeño Innovador (IDDI)}

Dado que los índices planteados en la literatura requieren de información específica y comparable sobre ciertas variables, y que las encuestas de innovación disponibles para el presente estudio no ofrecen los datos con las características requeridas para este tipo de análisis, se propone la construcción de un índice a partir de datos disponibles en ellas, basado en la metodología del Índice de Valor de Importancia Relativizado (IVI) - indicador adimensional que muestra el grado de importancia estadística que tiene un individuo en un grupo o contexto, según la totalidad de las características de las variables estudiadas utilizando el Análisis de Componentes Principales ACP- propuesta y aplicada a un proceso etnobotánico por (Lajones \& Lema, 2000). En este caso, se trata del Índice de Desempeño Innovador (IDDI) que se construye sobre la taxonomía de innovación propuesta por el Manual de Oslo según el cual, las componentes que describen la innovación en la industria son: innovación de producto, de proceso, de mercado, organizacional, y para los países en desarrollo, existe una categoría que se denomina innovación potencial que se refiere a aquellos esfuerzos en innovación que aún no han mostrado resultados (OECD, 2005).

El Índice de Desempeño Innovador IDDI puede calcularse para cada componente de innovación, o como valor promedio de todas las componentes, mostrando el porcentaje de desempeño innovador en los subsectores estudiados, o el potencial de innovación de las empresas en la muestra encuestada. Se calcula mediante varios procesos, el primero de los cuales consiste en determinar las variadas de orden superior que puedan desagregarse en otras en varios niveles, hasta llegar a las variables finales, refiriéndose a los diferentes tipos de innovación según taxonomía del Manual de Oslo (OECD, 2005) -innovación de productos, de procesos, de organización, de mercado e innovación potencial-. 
Luego, se seleccionan las variables del fenómeno que componen cada variada, las cuales se ponderan con algún criterio pertinente por parte de los investigadores o la asignación de puntajes de prioridad o ponderadores por paneles de expertos, desde las variables hasta las variadas de orden superior, que luego se relativizan según los pesos asignados. Se continúa después con un proceso de disminución de dimensionalidad del sistema para explicar su variabilidad, mediante componentes principales (ACP) aplicado desde cada variada de orden inferior, hasta las de orden superior en la totalidad del sistema resultante, con el fin de identificar colinealidades entre las variables y eliminar las redundancias entre ellas, es decir, desestimar aquellas variables que ofrecen información que ya se encuentra en el sistema y que introducen ruido. Con las variables encontradas se calcula el IVI para cada variada de orden inferior, como la sumatoria de los valores relativizados. A continuación se calcula el IVI de cada variada de siguiente orden, como la suma ponderada de los IVI de cada variada, multiplicada por el peso asignado de la variada correspondiente, y dividido por la sumatoria de los IVI de las subvariadas que componen la variada, como se muestra en la ecuación (1).

$$
I V I=\frac{\sum x_{i} P_{i}}{\sum P_{j}}
$$

Donde:

$x_{i}=$ valor de la variable luego de haberse convertido a escala de valores

$P_{i}=$ peso asignado por los expertos a la variable con respecto al fenómeno estudiado

$P_{j}=$ peso de la variada, compuesto por la sumatoria de los pesos de las variables que la componen

Con los valores resultantes de IVI para cada observación se conforman los IVI totales o IVITO, los cuales, se someten luego a un análisis de conglomerados (clúster) según varios métodos o estrategias de fusión y así se clasifica cada observación según al clúster al que pertenezca. Mediante el IVI$\mathrm{TO}$, se realizan comparaciones entre las diferentes encuestas y se identifican aquellos casos de mayor relevancia para el sistema. En caso en que el nivel de agregación de los datos de las variables sea superior, se toman los valores del IVITO en el nivel correspondiente y se calcula su valor promedio, el cual corresponderá al IDDI que se busca. El método de cálculo de los IVI e IVITO se empleó inicialmente en clasificaciones etnobotánicas, en las cuales se considera que una buena percepción de los fenómenos altamente aleatorios se logra con una explicación de variabilidad superior al $30 \%$, es decir, que al obtener componentes cuyos porcentajes de varianzas sean superiores a este valor, se considerarán suficientes para seleccionar dichas combinaciones lineales (Ter-Braak et al., 2002, citado en Lema, 2005). En vista de sus potencialidades, este es el método que se utiliza para el IDDI en el presente trabajo.

Cuando se calculan índices a partir de fuentes de datos con diferencias en estructura, diseño y contenidos como es el caso del presente estudio, se establece la relación que pueda existir entre tales índices mediante Análisis de Regresión Múltiple. Para establecer la consistencia del modelo que relaciona dos índices generados a partir de dos encuestas diferentes con diversas variables agrupadas en variadas con igual nombre, surgidas de un ACP, se debe verificar la hipótesis de que las variables del modelo solo dependen de elementos constitutivos del índice calculado (IDDI) y no de las variables accesorias que lo rodean, para lo cual se acude también al método de Modelos Lineales Generalizados.

\section{Objetivos y metodología}

Se propone la construcción de un Índice que permita conocer el desempeño innovador de la industria colombiana a nivel subsectorial a partir de los resultados de la primera y segunda encuestas de Innovación realizadas en Colombia en los años 1996 y 2005, que considere tanto las limitaciones de los datos, como la eliminación de colinealidades o redundancia de información en las variables seleccionadas.

La metodología para elaborar dicho índice consta de siete fases: 
Gráfica 1. Metodología ${ }^{1}$

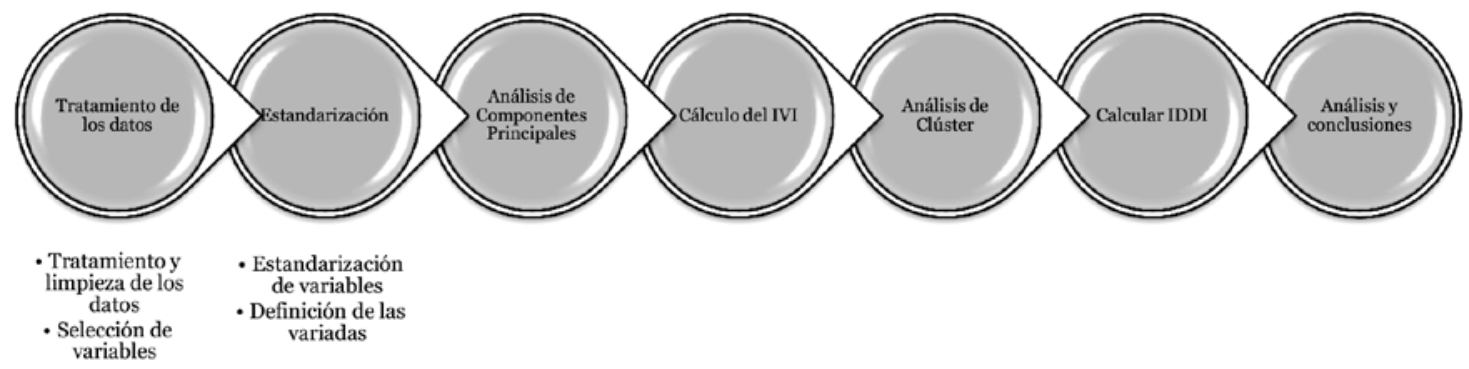

- Análisis de la información disponible en las encuestas para identificar variables con valores erróneos; una vez depurados los datos, se definen las variadas según los elementos de entrada, de proceso y de salida que indican la realización de esfuerzo o inversión en alguna de las actividades de innovación y desarrollo tecnológico, de acuerdo con los tipos de innovación a medir, mediante la pregunta ćcuál es la importancia que tienen las variables $(x 1, x 2, . ., x n)$ en la innovación de productos, de procesos, organizacional, de mercado y potencial? ponderando así las variables seleccionadas según su capacidad para describir el tipo de innovación respectiva.

- Debido a la variedad de escalas de medición y pesos de las diferentes variables, y para evitar problemas de conmensurabilidad por diferencias de magnitudes de las variables, se debe realizar un proceso de estandarización (Lema, 2005).

- Análisis de Componentes Principales a cada variada para reducir la dimensionalidad del sistema de variables. El software utilizado para determinar aquellas variables que no presentan multicolinealidades es Statgraphics ${ }^{\circledR}$.

- Cálculo del IVI como la sumatoria de los valores estandarizados para cada empresa y el índice total IVITO como la suma ponderada de los IVI.

- Realizar un Análisis de Clúster seleccionando la distancia euclídea al cuadrado y siete métodos de agrupamiento para determinar cuatro gru- pos de establecimientos productivos según valor del IVI total o IVITO y calcular el intervalo de confianza.

- Calcular tanto para el año 1996 (EDT1), como para 2005 (EDT2), el valor promedio ponderado del IVITO para cada subsector industrial el cual, se denomina Índice de Desempeño Innovador (IDDI).

- Búsqueda de la relación que pueda existir entre los índices calculados a partir de dos encuestas diferentes con diversas variables agrupadas en variadas con igual nombre, surgidas del ACP.

\section{Resultados}

\subsection{Cálculo del IDDI para la primera encuesta de innovación}

A continuación se presentan los resultados del procesamiento de las variadas mediante ACP para construir el Índice de Valor de Importancia con el cual se determina el IDDI para la encuesta de 1996. A manera de ejemplo se describe el cálculo de la variada sobre innovación de productos, y se presentan los resultados de las demás variadas.

\subsubsection{Variada sobre innovación de productos}

La variada sobre innovación de productos en la EDT1 se conformó inicialmente con 17 variables. Luego del primer ACP en el que se consideraron 885 casos completos, la dimensionalidad se redujo a seis

1 Fuente: Elaboración propia. 
variables con una explicabilidad del $56,37 \%$ con dos componentes principales, como lo muestra la Tabla 1. Las seis variables se relacionaron con:

- Tipos de innovación de productos: producto nuevo asociado con proceso productivo nuevo.

- Tipos de innovación de productos: producto nuevo para el mercado nacional pero existe en el internacional.

- Tipos de innovación de productos: producto nuevo para el mercado nacional $e$ internacional.

- Existencia de un departamento de control de calidad.
- Realización de actividades de diseño.

- Desarrollo de proyectos de I+D en el período evaluado.

Luego de calcular los valores propios de las componentes, los porcentajes de varianzas explicadas por cada una de éstas, los diferentes pesos de las variables en las dos componentes principales, así como la gráfica que muestra las componentes que tienen valores propios superiores a 1,0 y la distribución de las variables en el plano de las componentes principales, los resultados se observan en la Tabla 1.

Tabla 1. ACP de la variada sobre innovación de productos en la EDT12

Principal Components Analysis
\begin{tabular}{|c|c|c|c|}
\hline $\begin{array}{c}\text { Component } \\
\text { Number }\end{array}$ & Eigenvalue & $\begin{array}{c}\text { Percent of } \\
\text { Variance }\end{array}$ & $\begin{array}{c}\text { Cumulative } \\
\text { Percentage }\end{array}$ \\
\hline 1 & 2,28883 & 38,147 & 38,147 \\
\hline 2 & 1,09353 & 18,226 & 56,373 \\
\hline 3 & 0,774032 & 12,901 & 69,273 \\
\hline 4 & 0,649526 & 10,825 & 80,099 \\
\hline 5 & 0,608738 & 10,146 & 90,244 \\
\hline 6 & 0,585341 & 9,756 & 100,000 \\
\hline
\end{tabular}

Table of Component Weights

\begin{tabular}{|c|c|c|}
\hline & Component 1 & Component 2 \\
\hline Vi600A & 0,448495 & $-0,191329$ \\
\hline IV402_03 & 0,29074 & 0,621057 \\
\hline IV403_02 & 0,480324 & 0,067118 \\
\hline IV403_03 & 0,323275 & 0,555002 \\
\hline IV411 & 0,414651 & $-0,432909$ \\
\hline V501 & 0,455157 & $-0,278819$ \\
\hline
\end{tabular}

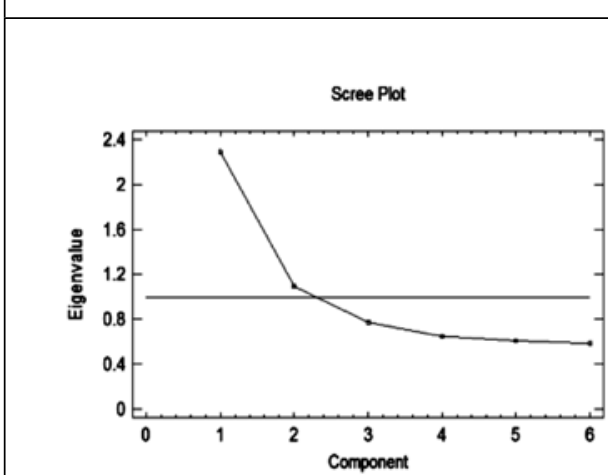

Diagrama de Criba

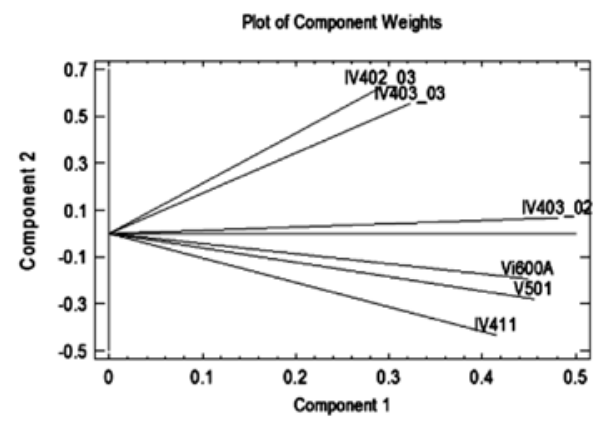

Gráfica de pesos de las componentes

2 Fuente: Cálculos propios a partir de la EDT1. 


\subsubsection{Las demás variadas de la EDT1}

El mismo procedimiento se llevó a cabo con las variadas de Innovación de Procesos, de Mercado, Organizacional, e Innovación Potencial. La reducción de dimensionalidad para las demás variadas estuvo entre el $10 \%$ y el $65 \%$ y el grado de explicabilidad luego de someterlas a los correspondientes ACP estuvo entre el $53 \%$ y el $67 \%$.

\subsubsection{Cálculo del IVITO para 1996}

Las variables resultantes del ACP en cada subsector industrial se estandarizaron para calcular tanto los IVI de cada variada, como el IVITO y el valor promedio ponderado de este por subsector, que corresponde al IDDI para el año 1996 (IDDI-96). Sólo se consideraron 743 empresas que tenían el tamaño identificado (A, B, C o D) y se obtuvieron los resultados de la Tabla 2.

Tabla 2. Valores del IDDI-96 y sus componentes ${ }^{3}$

\begin{tabular}{|c|c|c|c|c|c|c|}
\hline \multirow{2}{*}{ Subsector } & \multicolumn{5}{|c|}{ Componentes del IDDI- Promedios ponderados } & \multirow{2}{*}{ IDDI-96 } \\
\cline { 2 - 6 } & IVIProd & IVIProc & IVIOrg & IVIMerc & IVIPot & \\
\hline AlimByTb & 0,09 & 0,12 & 0,20 & 0,29 & 0,21 & 0,16 \\
\hline TxtConfCr & 0,06 & 0,08 & 0,16 & 0,16 & 0,15 & 0,11 \\
\hline MadPrdts & 0,06 & 0,04 & 0,13 & 0,12 & 0,12 & 0,08 \\
\hline Papellmprt & 0,07 & 0,10 & 0,18 & 0,15 & 0,16 & 0,11 \\
\hline Qcos & 0,11 & 0,10 & 0,19 & 0,24 & 0,24 & 0,15 \\
\hline Minometal & 0,10 & 0,10 & 0,16 & 0,19 & 0,20 & 0,13 \\
\hline MetalBas & 0,10 & 0,10 & 0,19 & 0,18 & 0,20 & 0,14 \\
\hline Pdtmetalymaq & 0,09 & 0,10 & 0,18 & 0,20 & 0,21 & 0,14 \\
\hline
\end{tabular}

Posteriormente, se hizo un análisis de clúster, y se obtuvieron cuatro agrupamientos del IVITO en orden descendente. El comportamiento de las componentes del IVITO y del IDDI-96 por cada subsector y tamaño de empresa en los agrupamientos G1 a G4 se observa en la Gráfica 2.

\subsection{Cálculo del IDDI a partir de la EDT2}

Para el cálculo de los IVI de la EDT2 se revisaron los diferentes archivos de las bases de datos y se seleccionaron 68 variables que conformaron las variadas sobre innovación de productos, de procesos, de organización, de mercado e innovación potencial. Se relacionaron los datos de las variables con los códigos de identificación de las empresas, pues eran diferentes en cada archivo de datos y se identificaron los valores erróneos de datos en las diferentes variables.
Los valores categóricos se transformaron a escalas ascendentes de 0 a 3 según el grado de importancia, siendo 3 el valor correspondiente a muy importante. Las respuestas afirmativas y negativas se convirtieron a 1 y 0 , respectivamente. Finalmente, se procesaron 3.980 de 4.927 casos completos.

Como resultado, se seleccionaron 19 variables sobre innovación de producto, 21 sobre innovación de procesos, 12 sobre innovación organizacional y 12 variables relacionadas con inversiones que los encuestados dicen haber dirigido al mercadeo. Para el análisis de la innovación potencial se recurrió al archivo de datos de la EDT2 sobre los objetivos de la innovación en el período 2003-2004 y se seleccionaron cuatro variables. En total se seleccionaron 68 variables que, luego de ser sometidas a un ACP, se redujeron a 15 .

3 Fuente: Cálculos propios a partir de la EDT1. 
Gráfica 2. Valores del IDDI en el año 1996 y sus componentes (IVI) por cada agrupación G1 a G4 en los diferentes subsectores $^{4}$

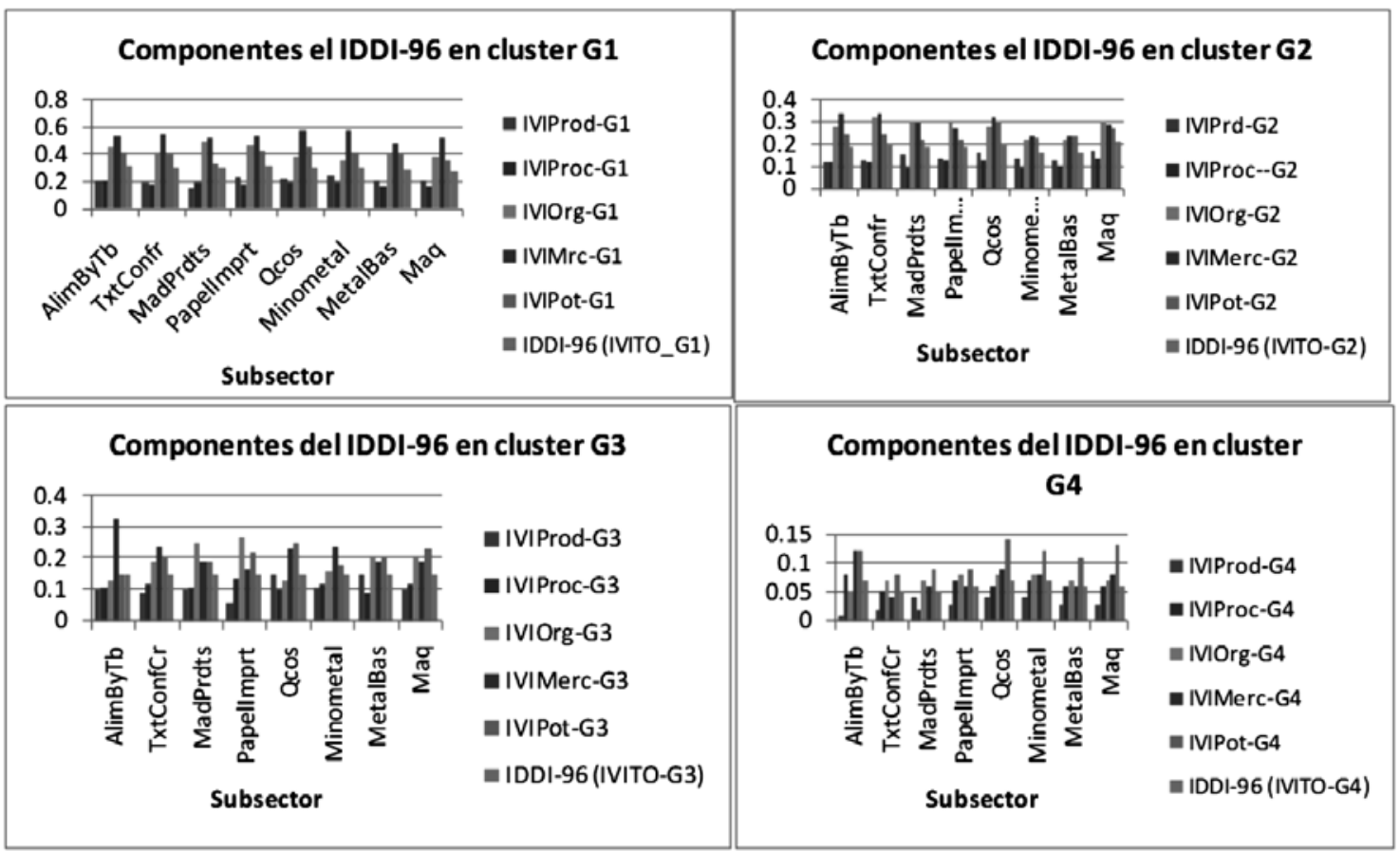

Los IVI de cada tipo de innovación que componen el IDDI para cada subsector en el año 2005, calculados a partir de la EDT2, se muestran en la Tabla 3.
Los IVITO de los ocho subsectores reflejaron comportamientos diferentes según el tamaño de las empresas. Con el fin de visualizar esta situación, los va-

Tabla 3. Valores del IDDI-05 y sus componentes ${ }^{5}$

\begin{tabular}{|c|c|c|c|c|c|c|}
\hline \multicolumn{7}{|c|}{ Componentes del IDDI $^{*}$} \\
\hline Subsector & IVIProd & IVIProc & IVIOrg & IVIMerc & IVIPot & IDDI-05 \\
\hline AlimByTb & 0,03 & 0,05 & 0,02 & 0,02 & 0,17 & 0,04 \\
\hline TxtConfCr & 0,04 & 0,02 & 0,01 & 0,02 & 0,18 & 0,04 \\
\hline MadPrdts & 0,05 & 0,03 & 0,01 & 0,02 & 0,19 & 0,04 \\
\hline Papellmprt & 0,02 & 0,04 & 0,01 & 0,02 & 0,20 & 0,04 \\
\hline Qcos & 0,05 & 0,05 & 0,01 & 0,02 & 0,20 & 0,05 \\
\hline Minometal & 0,06 & 0,05 & 0,03 & 0,02 & 0,18 & 0,05 \\
\hline MetalBas & 0,04 & 0,05 & 0,01 & 0,02 & 0,19 & 0,04 \\
\hline Pdtmetalymaq & 0,08 & 0,06 & 0,01 & 0,02 & 0,18 & 0,06 \\
\hline
\end{tabular}

Valores promedios ponderados por tamaño de empresa.

4 Fuente: Elaboración propia a partir de la EDT1.

5 Fuente: Cálculos propios a partir de la EDT2. 
lores del IVITO ponderado por tamaño de empresa para los años 1996 y 2005 se ilustran en la Gráfica 3, donde se identifican como IVITO-96 e IVITO-05 respectivamente, y los tamaños de las empresas con letras mayúsculas entre la A y la D (Gráfica 3).

También se calcula el IDDI en el año 2005 (IDDI-05) correspondiente a las cuatro agrupaciones o clúster resultantes del IVITO calculado de manera descendente, observándose un comportamiento similar en todas las componentes por subsector industrial. Los resultados y el análisis detallado se presenta en Manrique (2012).

El comportamiento comparado de los IDDI a partir de las EDT1 y EDT2 para cada subsector industrial colombiano se ilustra en el Gráfica 4.

En la Gráfica 4 se observa cómo el desempeño innovador de los subsectores industriales colombianos fue superior en 1996 al de 2005, y que los subsectores de Alimentos, Químicos, Metales básicos y Maquinaria y equipos tuvieron el desempeño más alto de todos los demás subsectores en 1996.

\subsection{Relaciones entre el IDDI-96 y el IDDI-05}

Para establecer relaciones entre los dos índices calculados, se realizó un análisis de regresión múltiple entre los IDDI de 1996 y 2005 y sus diferentes com- ponentes, según los datos de las Tablas 2 y 3. Las únicas relaciones significativas se encontraron entre el componente de innovación organizacional del año 2005 en función de todas las componentes del IVITO96.

No obstante los resultados obtenidos por regresión múltiple, para establecer la consistencia del modelo generado en dos encuestas con diversas variables agrupadas en variadas con igual nombre, surgidas del Análisis de Componentes Principales, se acudió al método de Modelos Lineales Generalizados. Previo al sometimiento al proceso descrito, para poder verificar las relaciones en el comportamiento de las encuestas madre, las cuales se habían procesado por subsector y por tamaño de empresa, se procedió a elaborar un modelo de reclasificación de una de las variables, tomando para ello el número de las empresas para el año 2005 por medio de una variable cualitativa para predecir en el año 2005 el equivalente a alguna de las variables del año 1996 (como el número de empresas, por ejemplo) para someterlo, posteriormente, a un modelo lineal generalizado mediante los siguientes rangos: $\mathrm{A}<=$ 10 empresas; $10<\mathrm{B}<=20$ empresas; $20<\mathrm{C}$ $<=30$ empresas y $30<\mathrm{D}$ empresas. Con el modelo ajustado, se reclasificaron los tamaños de las empresas de ambas encuestas. Sometida a análisis esta base de datos conjunta para predecir el IDDI, se encontró un modelo en el que efectivamente se

Gráfica 3. Valor del IVITO por subsector y tamaño para los años 1996 y $2005^{6}$
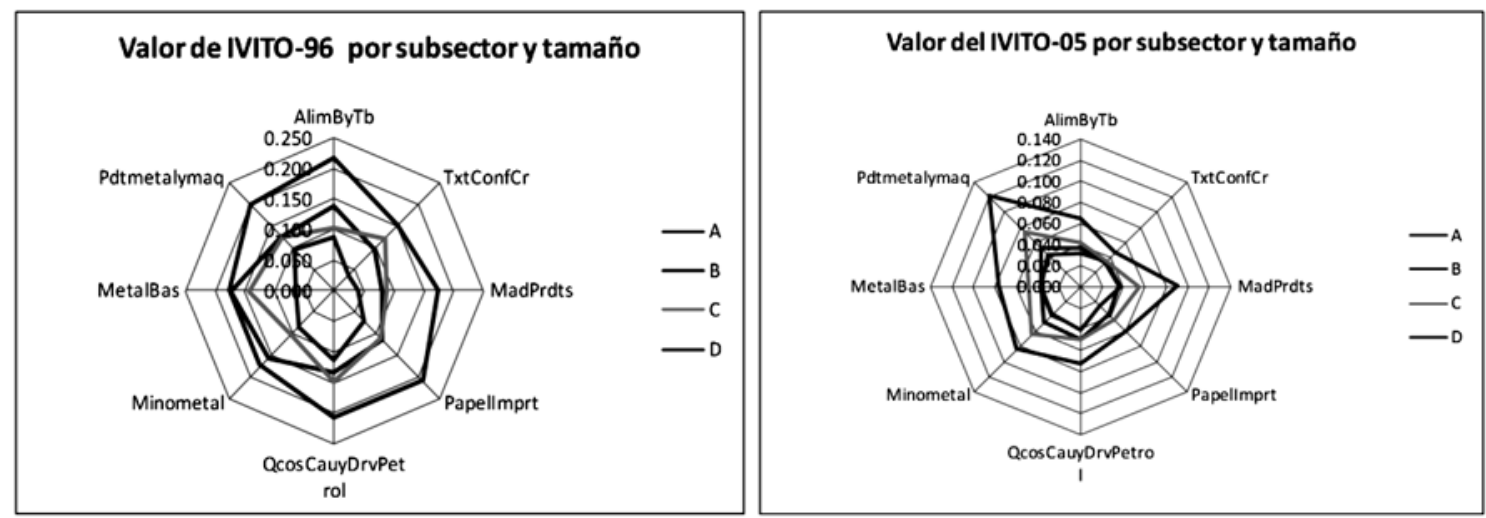

6 Fuente: Elaboración propia a partir de la EDT1 y la EDT2. 
Gráfica 4. IDDI en los años 1996 y 2005 por subsector industrial ${ }^{7}$

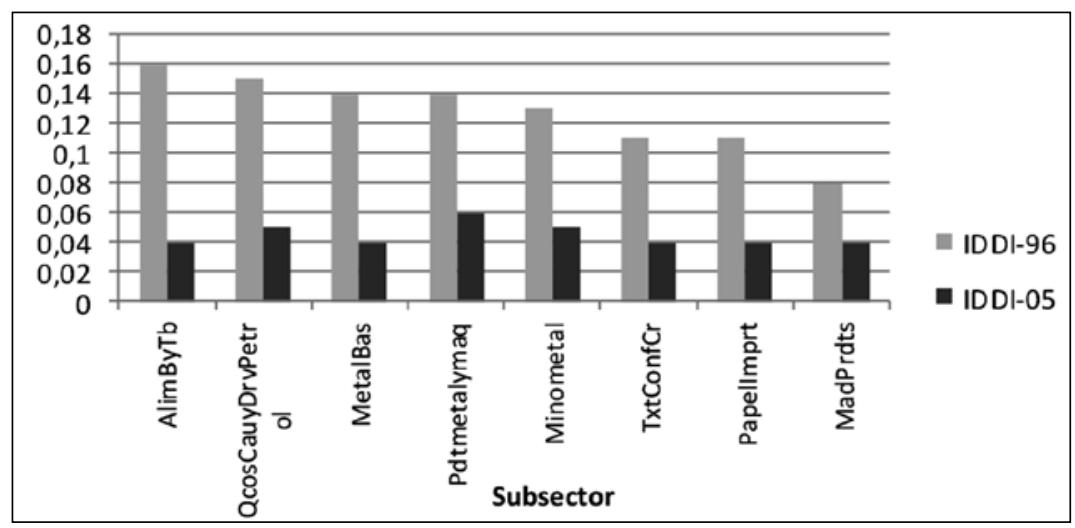

determinó que las variables categóricas, tamaño de las empresas, número de empresas y grupo, no resultaban significativas, comprobando la hipótesis nula sometida a verificación, excepto para el año de la encuesta, como era de esperarse, por otros análisis ya mostrados.

El Análisis de Varianza (ANAVA) para el modelo matemático ajustado mostró estadísticos favorables, descartándose presencia de multicolinealidad, encontrándolo aplicable en cualquier circunstancia que disponga de las variables explicativas que lo ajustan (Manrique, 2012). El ajuste relativo entre los valores observados vs ajustados mediante el modelo calculado se observa en la Gráfica 5.
Independiente del año en que se desee evaluar lo sucedido y con el ajuste que permitió la información disponible, se llegó al modelo de la ecuación (2).

$$
\begin{gathered}
\text { IDDI }=-0,0111576+0,657279 I V I P r o d+ \\
0,782665 \text { IVIProc }
\end{gathered}
$$

En este modelo, calculado con límites de confianza del 95\% para los coeficientes, se observa que el IDDI para cualquier año en el rango estudiado, se relaciona directamente con el desempeño innovador de productos y el de procesos. El IDDI calculado como valor promedio ponderado de las empresas en los subsectores para 1992 y 2006, y el IDDI modelado se aprecian en la Gráfica 6.

Gráfica 5. Cuantiles para valores observados vs ajustados por el modelo ${ }^{8}$

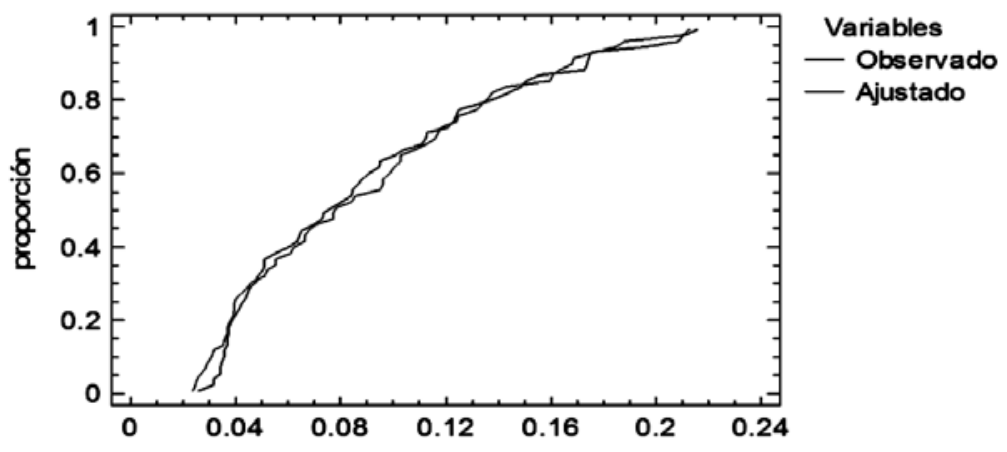

Fuente: Cálculos propios a partir de la EDT1 y EDT2.

Fuente: Cálculos propios. 
Gráfica 6. IDDI promedio ponderado por subsector vs IDDI modelado para 1996 y $2005^{9}$

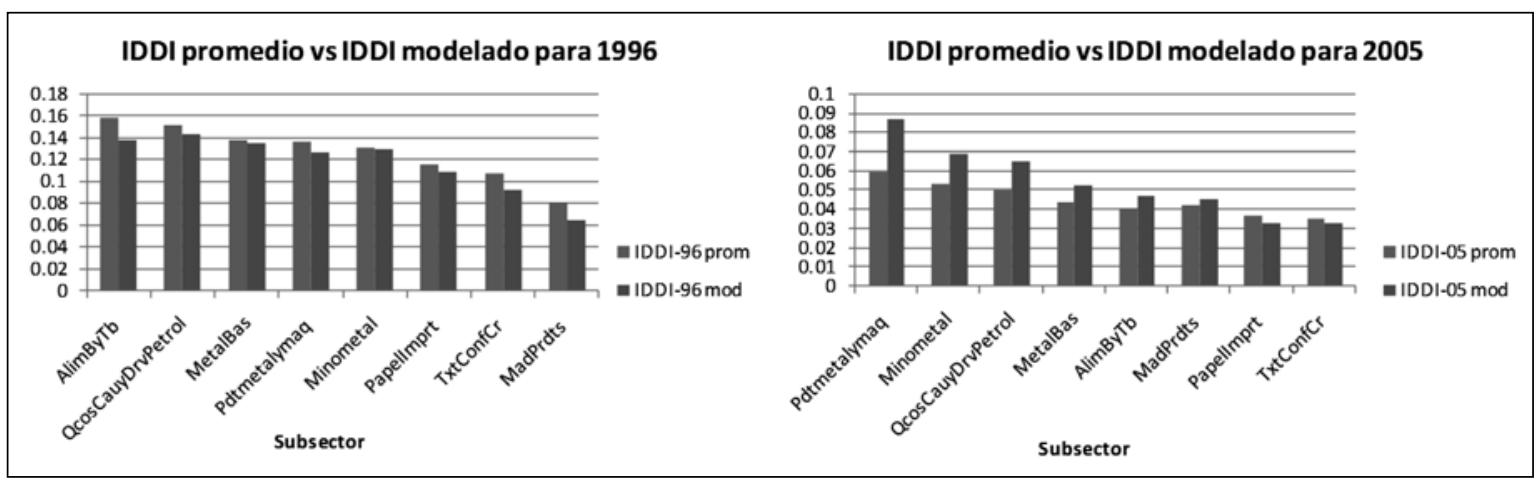

Los subsectores industriales que presentaron un mayor IDDI fueron, en su orden, los de Químicos, Alimentos, Metales básicos, Minerales no metálicos y Maquinaria, y los componentes relacionados con la innovación de mercado, potencial y de organización presentaron valores más altos en los IVI para todos los subsectores. Se destaca especialmente el IVI de la innovación de mercado en el subsector de alimentos, mientras que los componentes de producto y de proceso fueron más bajos en todos los subsectores.

\section{Análisis de resultados}

El índice calculado se compone de cinco variadas relacionadas con los tipos de innovación mencionados en el Manual de Oslo: Innovación de productos, innovación de procesos, innovación organizacional, innovación de mercado o comercial $y$, finalmente, innovación potencial. El promedio ponderado de las variadas por cada subsector, según el tamaño de las empresas, se denomina Índice de Desempeño Innovador IDDI.

Los resultados del cálculo del índice se interpretan mediante una escala que indica el porcentaje de innovación realizada: $(0 \%)$ cuando no hay innovación, (1 a 25\%) cuando la innovación es incipiente, (>25$50 \%$ ) cuando hay baja innovación, ( $>50-75 \%)$ para innovación media, y (>75-100\%) cuando el nivel de innovación es alto.
La búsqueda de relaciones significativas entre los IDDI de las dos encuestas y sus componentes se realizó mediante Análisis de Regresión Múltiple y mediante el método de Modelos Lineales Generalizados. Este último método resultó tener más consistencia para relacionar las variables de interés entre las encuestas y permitió establecer que, el Índice de Desempeño Innovador (IDDI) para cualquier año en el rango estudiado se relacionaba directamente con la innovación de productos y la innovación de procesos.

Los resultados muestran que, según la Encuesta del año 1996, el valor más alto del IDDI lo obtuvo el subsector de Alimentos $(0,16)$, mientras que el más bajo fue para el subsector de Maderas $(0,08)$. Para este año, las componentes innovación de producto $e$ innovación de proceso, aunque eran las que más sobresalían en todos los subsectores, eran deficientes y no superaban el valor de 0,30 , siendo los subsectores de Químicos, Alimentos y Maquinaria los de mejor desempeño. Esto indica que, en su mayor parte, las empresas sólo realizaban cambios marginales en innovación, dirigidos especialmente a las mejoras en los aspectos, organizacional y de mercado.

Para el año 2005, los valores promedios ponderados por subsector oscilaban entre 0,04 y 0,06, resultando muy inferiores a los IDDI de 1996, lo que, para 2005 , indica que la industria colombiana había dis-

9 Fuente: Cálculos propios. 
minuido considerablemente su actividad innovadora en todos los frentes.

El comportamiento de las cinco componentes de innovación también fue diferente en 2005 pues, la principal característica esta vez fue el bajo valor de los índices, siendo las innovaciones de mercado y organizacional las más bajas con valores inferiores a 0,05 . Por otro lado, se destaca la importancia que adquirió la innovación potencial (entre 0,15 y 0,20) relacionada con los proyectos y actividades de $\mathrm{I}+\mathrm{D}$ que todavía no habían mostrado resultados en ningún subsector.

\section{Conclusiones}

Según el Manual de Oslo (OECD, 2005), la disponibilidad de datos procesados y limpios es una de las principales limitantes que tienen los investigadores para efectuar propuestas que conduzcan al análisis de la innovación en países en desarrollo. En Colombia, los datos de las dos primeras Encuestas de Innovación realizadas en los años 1996 y 2005 presentan características diferentes por efecto de diseño y aplicación que no permiten su comparabilidad de manera directa. En el trabajo acá reportado, los datos se sometieron a procesos de limpieza y adecuación para su respectivo análisis y, además, a un Análisis de Componentes Principales con el cual se verificó la existencia de información redundante.

Debido a las limitaciones de las fuentes de datos, en el caso colombiano no es posible aplicar índices de innovación que exijan una alta consistencia en la definición y la métrica de las variables involucradas. Para obviar esta dificultad, se propone en este trabajo el cálculo de un nuevo Índice de Desempeño Innovador (IDDI) construido a partir de las encuestas de innovación realizadas en 1996 y 2005, utilizando una metodología que tolera una considerable heterogeneidad en las variables y métricas de dichas encuestas, y que se basa en la metodología del índice de Valor de Importancia Relativizado de Lajones \& Lema (2000).

El desempeño innovador de la industria a partir del promedio del Índice de Desempeño Innovador en el año 1996 se situó entre 8\% y 16\%, considerado como incipiente en la escala de medición. Los resultados de 2005 mostraron valores entre $4 \%$ y $6 \%$ situados en el rango de innovación incipiente e inferiores a los de 1996, coincidiendo con lo reportado por (Manrique \& Velásquez, 2012).

En el año 1996, los subsectores de mejor desempeño innovador fueron el de Alimentos, Químicos, Metales básicos y el de Maquinaria, y los de menor desempeño fueron los de Textiles y Maderas, con un valor máximo de 0,16 y un mínimo de 0,08. Las componentes de innovación de producto e innovación de proceso fueron los que más sobresalieron en todos los subsectores, con valores máximos de 0,30. Para 2005, los valores por subsector oscilaron entre 0,04 y 0,06 , y los subsectores sobresalientes fueron el de Maquinaria, Materiales no metálicos y el de Químicos, mientras que los de menor desempeño fueron el de Papel e imprentas y el de Textiles nuevamente. Esta vez, la innovación potencial (entre 0,15 y 0,20 ) fue la componente más importante, relacionada con los proyectos y actividades de $\mathrm{I}+\mathrm{D}$ que todavía no habían mostrado resultados en ningún subsector.

Aunque el comportamiento del IDDI del año 2005 por tamaño de empresa es superior en las grandes para la mayoría de subsectores, la poca cantidad de estas empresas con respecto al total hace que el promedio de los índices sea reducido. Posiblemente, si los análisis se realizaran a niveles inferiores de desagregación de los datos, los índices podrían mostrar de manera más apropiada el comportamiento de las variables involucradas.

Por otro lado, la existencia de un gran número de empresas potencialmente innovadoras puede sugerir la presencia de serias barreras a la innovación o de tentativas prematuras al respecto con ausencia de los recursos y las capacidades necesarias, lo cual prioriza la importancia de las actividades sobre los resultados.

El índice de Desempeño Innovador IDDI aquí propuesto, calculado con base en el Índice de Valor de Importancia Relativizado IVI, muestra grandes potencialidades para la determinación del desempeño innovador de las industrias o grupos industriales 
pues, en primer lugar, reduce la redundancia en la información de las diferentes variables lo cual, es muy pocas veces aplicado en el cálculo de los diferentes índices y puede influir considerablemente en los resultados. Adicionalmente, puede ser una alternativa importante para el procesamiento de datos y encuestas con las características mencionadas, que no pueden enfrentarse con otras metodologías más comunes en estos casos.

Con base en el procesamiento de la información disponible y los resultados de este estudio, es recomendable tener en cuenta para el diseño de futuras encuestas de innovación, la utilización continua en el tiempo de variables similares que permitan la medición de los diferentes tipos de innovación con base en los diferentes índices internacionales propuestos y sus componentes a utilizar en los análisis.

\section{Referencias}

Archibugi, D. \& Coco, A. (2004). A new indicator of technological capabilities for developed and developing countries (ArCo). En: World Development, 32(4): 629-654.

Archibugi, D. \& Pianta, M. (1996). Measuring technological change through patents and innovation surveys. En: Technovation, 16(9): 451-468.

Blackman, A., Seligman, E. \& Sogliero, G. (1973). An innovation index based on factor analysis. En: Technological Forecasting and Social Change, 4(3): 301-316.

Buesa, M., Heijs, J., Martínez Pellitero, M. \& Baumert, T. (2006). Configuración estructural y capacidad de producción de conocimientos en los sistemas regionales de innovación: un estudio de caso español. Instituto de Análisis Industrial y FinancieroIAIF, Universidad Complutense de Madrid, Documento de trabajo, $\mathrm{N}^{\circ} 45$.

Buesa, M., Navarro, M., Molero, J., Aranguren, M. \& Olarte, F. (2001). Indicadores de la ciencia, la tecnología y la innovación: metodología y fuentes para la CAPV y navarra. Eusko Ikaskuntza, San Sebastián.

Cardona, M. \& Cano, C. (2005). Innovación empresarial y capacidades tecnoproductivas: análisis sectorial y regional en Colombia en el período 1990-2002. Medellín.

Chaves Castro, Á. (2005). Un modelo de cointegración estacional de la producción industrial, Colombia 1993-2005. En: Cuadernos de Economía, 24 (43): 105-128.

Delgado, J. \& Sánchez, G. (1999). Caracterización de las ramas industriales en España según su comportamiento innovador. En: Ekonomiaz - Revista Vasca de Economía, (44: 256-277.

Dutta, S., Ullah Khan, A., Bharadwaj, A. \& Kaul, T. (2008). Global innovation index 2008-2009: more on methodology - management today. Disponible en: http://www.managementtoday. co.uk/news/610009/.

Fagerberg, J. \& Srholec, M. (2005). Catching up: what are the critical factors for success? (UNIDO World Industrial Development
Report 2005). Norway: Centre for Technology, Innovation and Culture, University of Oslo.

Fagerberg, J., Srholec, M. \& Knell, M. (2007). The competitiveness of nations: why some countries prosper while others fall behind. En: World Development, 35(10): 1595 - 1620.

Green, S. G., Gavin, M. B. \& Aiman-Smith, L. (1995). Assessing a multidimensional measure of radical technological innovation. En: Engineering Management, IEEE Transactions on, 42(3): 203-214.

He, J. \& Fallah, M. (2011). The typology of technology clusters and its evolution - Evidence from the hi-tech industries. En: Technological Forecasting and Social Change, 78(6): 945-952.

Lajones D. \& Lema, Á. (2000). Propuesta de un índice de valor de importancia aplicado a la etnobotánica de tres comunidades en el Ecuador. Universidad Nacional de Colombia- Sede Medellín, Medellín.

Langebaek, A. \& Velásquez, D. (2007). Determinantes de la actividad innovadora en la industria manufacturera colombiana. En: Borradores de Economía, Banco de la República de Colombia, (433): 1-33.

Lema, Á. (2005). Elementos de Estadística Multivariada (2a ed.). Medellín: Universidad Nacional de Colombia- Sede Medellín.

Leonardi, V., García, I. \& Cristiano, G. (2009). Desempeño innovador de un grupo de Mipymes agroindustriales argentinas. En: Economía y Sociedad, XIV: 45-64.

Lugones, G. \& Suarez, D. (2010). Science, technology and innovation indicators for policymaking in developing countries: an overview of experiences and lessons learned. Division of Technology and Logistics of UNCTAD.

Malaver, F. \& Vargas, M. (2006). Capacidades tecnológicas, innovación y competitividad de la industria de Bogotá y Cundinamarca. Resultados de una encuesta de innovación. Cámara de Comercio de Bogotá y Observatorio de Ciencia y Tecnología.

Manrique, J. (2012). El uso energético y la innovación en la industria colombiana a partir de encuestas oficiales. Unpublished Tesis Doctoral, Universidad Pontificia Bolivariana - Medellín.

Manrique, J. \& Velasquez, J. (2012). Innovation indices of the Colombian industrial groups from two national innovation surveys. Technology Management in the Energy Smart World (PICMET). Proceedings of PICMET (July 31-Aug. 42011): 1-10. Disponible en: http://ieeexplore.ieee.org/stamp/stamp.jsp?tp = \&arnumber $=6$ 017612\&isnumber $=6017605$

Martinez, M. \& Baumert, T. (2003). Medida de la capacidad innovadora de las comunidades autónomas españolas: construcción de un índice regional de la innovación. IAIF- Instituto de Análisis Industrial y Financiero- Universidad Complutense de Madrid: 1-62.

Molero Z., J. (2007). Informe crítico sobre la innovación tecnológica en la economía española: abriendo la "caja negra". En: Revista Madrid, $\mathrm{N}^{\circ} 40$. Tribuna de debate.

Mora Luna, A. \& Davidsen, P. (2006). An investigation of the innovation performance in the capital goods sector in Colombia: using the system dynamics approach. En: Revista de Dinámica de Sistemas, 2(1): 103-151.

OECD (2005). Oslo manual: the measurement of scientific and technological activities. Proposed guidelines for collecting and interpreting innovation data. Third edition. OECD Publications, Paris.

Okay, N., Konukman, A. \& Akman, U. (2009). Analysis of innovation and energy proles in the turkish manufacturing sector. Munich Personal RePEc Archive - MPRA. MPRA Paper No 16779: 1-18. 
Olaya, A. (2009). Los patrones de innovación tecnológica de las empresas españolas: su caracterización desde la dinamica sectorial mundial de invención. Unpublished Tesis Doctoral, Universidad CEU San Pablo, Madrid.

Pedraza, A., Cabrera, M., Duarte, M., Gutiérrez, M., Lamprea, P. \& Lozano, R. (2008). Visión general del inventario nacional de gases de efecto invernadero - Capítulo 1. Bogotá: IDEAM.

Reichstein, T. (2004). Does product innovation and firm growth go hand in hand? En: Research on Technological Innovation and Management Policy, 8: 343-367.

Restrepo, J. (2008). Capacidades de innovación tecnológica y el desempeño innovador empresarial en Colombia a partir de la Encuesta Nacional de Innovación de 2005. Paper presentado en el I Congreso Internacional de Gestión Tecnológica e Innovación, Bogotá.
RICYT, OEA, CYTED, COLCIENCIAS \& OCYT (2001). Manual de Bogotá. Normalización de indicadores de innovación tecnológica en América Latina y el Caribe.

Robledo Velásquez, J. (1997). The role of higher education institutions and the government in the industrial innovation process: the case of the Colombian capital goods industry. Ph.D. diss., University of Sussex, Brighton.

Salazar, M. \& Vargas, M. (1998). Encuesta sobre desarrollo tecnológico en la industria colombiana. Departamento Nacional de Planeación, Unidad de Desarrollo Empresarial, División de Desarrollo Tecnológico. Bogotá.

Sánchez, P. \& Castrillo, R. (2006). La tercera edición del manual de Oslo: cambios e implicaciones. Una perspectiva de capital intelectual, $\mathrm{N}^{\circ} 35$. Madrid. 